\title{
Type 1 neurofibromatosis complicated by pulmonary artery hypertension : a case report
}

\author{
Sara Simeoni', Antonio Puccetti ${ }^{2}$,Marco Chilosi ${ }^{3}$, Elisa Tinazzi', Daniele Prati ${ }^{4}$, \\ Roberto Corrocher', and Claudio Lunardi' \\ ${ }^{1}$ Section of Internal Medicine, Department of Clinical and Experimental Medicine, University of \\ Verona ; Institute G. Gaslini and Department of Experimental Medicine, University of Genova, Italy ; \\ ${ }^{3}$ Department of Pathology, University of Verona ; and ${ }^{4}$ Cardiology Unit, University Hospital, Verona, \\ Italy
}

\begin{abstract}
We describe the case of a patient with neurofibromatosis type 1 (NF1) complicated by severe pulmonary aterial hypertension (PAH) ; only seven cases have been reported on this association so far, and PAH seems to be related to the vascular involvement of neurofibromatosis. The histology of our patient's lung tissue showed thickening of arteries and veins by medial and/or intimal hypertrophy and fibrosis. In order to exclude a familiar PAH, the analysis of the bone morphogenetic protein receptor 2 gene was carried out, but no mutations were found.

On the basis of histological findings and of the results of genetic study we believe that PAH was a complication of NF1 in our patient and we suggest to screen patients with NF1 for the presence of PAH by means of trans-thoracic echocardiogram. J. Med. Invest. 54 : 354-358, August, 2007
\end{abstract}

Keywords : neurofibromatosis type 1, pulmonary artery hypertension, bone morphogenetic protein receptor 2

\section{INTRODUCTION}

Neurofibromatosis type 1 (NF1), also known as von Recklinghausen's disease, is an autosomal dominant disease with complete penetrance and extremely variable expression ; the incidence is approximately of 1 in 4000 live births (1). The predominant clinical hallmarks of NF1 are cafè-au-lait macules, neurofibromas, Lisch nodules of the iris and axillary freckling. Other clinical manifestations are abnormalities of the cardiovascular, gastrointestinal, renal and endocrine systems, facial and body disfigurement, cognitive deficit, and malignancies of the peripheral nerve sheath and central nervous system (2). The

Received for publication May 25, 2007 ; accepted July 18, 2007.

Address correspondence and reprint requests to Claudio Lunardi, $\mathrm{MD}$, Section of Internal Medicine, Department of Clinical and Experimental Medicine, University of Verona, Policlinico GB Rossi, Piazzale LA Scuro, 37134 Verona, Italy and Fax : +39-045580111.
NF1 gene on chromosome 17q11.2 spans more than $350 \mathrm{~kb}$ of genomic DNA and contains 60 exons (1). The NF1 gene product neurofibromin is a GTPase activating protein for members of p21ras (Ras) protein family. Since neurofibromin negatively regulates Ras output by accelerating the conversion of active Ras-GTP to inactive Ras-GDP, loss of neurofibromin function leads to downstream cell growth activation (3). Recent findings report that NF1 product modulates also protein kinase B (Akt), an important regulator of cell proliferation and survival (4).

Pulmonary artery hypertension $(\mathrm{PAH})$ is a disease of the small pulmonary arteries characterized by vessel proliferation and vessel wall remodelling. It results in a progressive increased in pulmonary vascular resistance and, ultimately in right ventricular failure and death. A diagnosis of primary (or idiopatic) pulmonary artery hypertension (PAH) is made when no known risk factors or other causes 
are identified (5). It is a progressive disease that usually leads to death within 5 years from the diagnosis.

Familial pulmonary hypertension is a rare ( $6 \%$ of all cases) autosomal dominant disorder with reduced penetrance, that has been mapped to a $3-\mathrm{cM}$ region on chromosome $2 q 33$. It is characterized by monoclonal plexiform lesion on proliferating endothelial cells. The disease can occur from infancy throughout life (6). Mutations in the gene encoding for the bone morphogenetic protein type II receptor (BMPR2) have been identified in $72 \%$ of affected families and $26 \%$ of apparently sporadic cases. The mutations are widely dispersed throughout the gene (7). BMPs are members of the transforming growth factor (beta) superfamily and affect intracellular signalling via Smads and mitogen-activated protein kinases (8).

We report here the case of a patient with von Recklinghausen disease complicated by severe PAH. The analysis of the BMPR2 gene did not show the presence of any mutation.

\section{CASE REPORT}

A 51-year-old Caucasian woman was admitted to our hospital because of progressive dyspnea on exertion and hyposthenia (NYHA class III). She had a history of hypertension since the age of 20.

Physical examination showed many cutaneous neurofibromas on her trunk, and multiple café au lait spots, the biggest one $(4 \mathrm{~cm})$ on her left wrist. She had neither peripheral or central cyanosis nor clubbing. Her blood pressure was 180/105 mm Hg and heart rate 110 beats/min ; there were inspiratory crackles at the right lung and a raised jugular venous pulse. Examination of the heart was normal, the liver was palpable at the costal margin and there was a swelling on the right clavicle region.

Laboratory investigations revealed high ESR (57 $\mathrm{mm} / \mathrm{Ih} ; \mathrm{nv}<35)$ and CRP $(37 \mathrm{mg} / \mathrm{L} \mathrm{nv}<5)$. Full blood count showed iposideremic microcytic anaemia (Hb 9,2 gr/dl, MCV 74 fl, MCH 24 pg). Autoantibodies were negative; liver and renal functions and coagulation tests were normal. Serologic testing for HIV was negative. Arterial blood gas tensions (on room air) were as follows : $\mathrm{PH} 7,43, \mathrm{PCO}_{2}$ $27 \mathrm{mmHg}, \mathrm{PO}_{2} 72 \mathrm{mmHg}, \mathrm{HCO}_{3} 18 \mathrm{mmol} / \mathrm{L}, 02$ Sat $90 \%$.

Neck ultrasonography showed a mass of $5 \mathrm{~cm}$ at the right sovraclavear region : the needle biopsy of the mass was not diagnostic. Chest X-ray showed bilateral pleural effusion ; chest computed axial tomography showed also two nodular lesions with irregular margins at the basal region of the right lung with pleural infiltration and a large mass in upper mediastinum. Bronchoscopy was negative. An abdomen ultrasonography was normal. A transthoracic echocardiogram revealed concentric hypertrophy of left ventricle, enlarged left atrium (48 $\mathrm{mm}$ ), interventricular septum of $13 \mathrm{~mm}$ and a right ventricular systolic pressure of $35 \mathrm{mmHg}$ (the peak of gradient through a mild tricuspid regurgitation was $30 \mathrm{mmHg}+5 \mathrm{mmHg}$ of right atrial pressure, by the normal size of a collapsing vena cava, were added to estimate right ventricular systolic pressure).

The patient was unable to carry out the spirometric test.

Biopsy of the mediastinum mass was compatible with schwannoma. The morphological examination of pulmonary tissue was consistent with pulmonary hypertension. The main histopathological features included the presence of focal areas of necrosis, the occurrence of numerous alveolar macrophages containing hemosiderin, and a diffuse alteration of vessel walls. Both arteries and veins appeared thickened by medial and/or intimal hypertrophy and fibrosis (Fig. 1). Infiltration of S100+ neurofibromatosis cells was not evident.

On the basis of the histopathological findings and of the worsening of dyspnoea, after two months we performed a second echocardiogram, that showed severe dilation of right chambers, with moderate tricuspid regurgitation, dilatation of vena cava and severe right ventricular systolic pressure $(115 \mathrm{mmHg})$.

A cardiac catheterization confirmed the presence of severe high PAH $(108 / 39 \mathrm{mmHg}$; mean, $65 \mathrm{mmHg}$ ), mean pulmonary wedge pressure 11 mmHg. After PGI2 infusive test (vasoreactivity testing), pulmonary vascular resistance remained unchanged.

Plasma N-terminal pro-B type natriuretic peptide (proBNP) level was 12,6 pmol/1 (nv < 14,8 pmol/L). However its levels markedly increased after three and nine months : 40,8 pmol/1 and $472 \mathrm{pmol} / 1$ respectively) ; cardiac troponin T was normal.

Since the patient's grandmother died of lung failure, the genetic analysis of BMPR2 was carried out in order to exclude a familiar PAH.

Based on the diagnosis of pulmonary hypertension, she was started on diuretics, warfarin and bosentan therapy (62,5 $\mathrm{mg}$ twice day for 4 weeks and then $125 \mathrm{mg}$ twice daily). NYHA class as well 

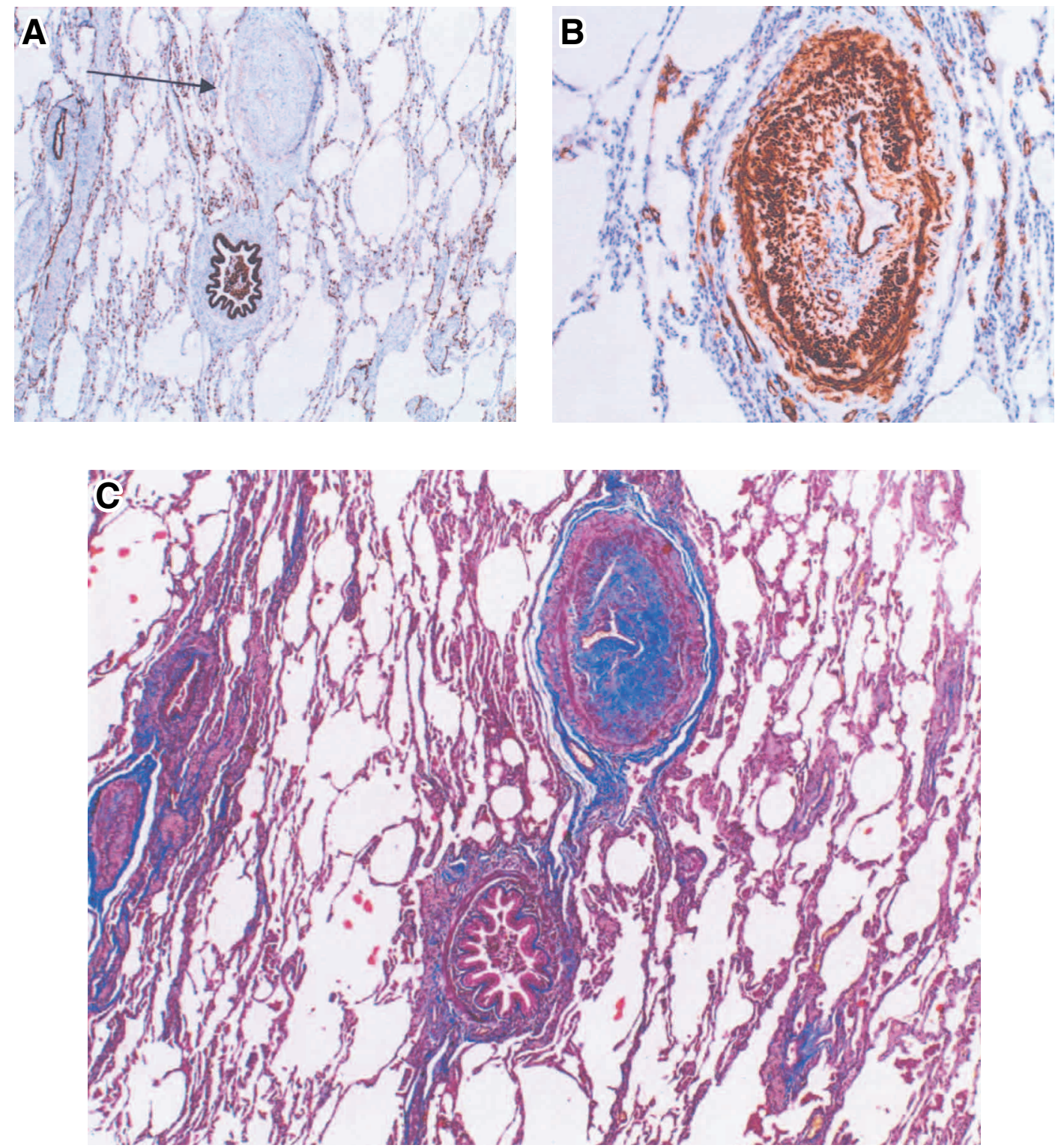

Fig. 1. Pulmonary artery with medial hypertrophy and marked intimal fibrosis.

(A) Anti-cytokeratin immunostaining (arrow). (B) Alpha-smooth-muscle actin and (C) trichrome staining.

as the 6 minutes walking test remained stable for nearly two and half years but recently, because of worsening of dyspnoea, sildenafil therapy has been introduced.

\section{Analysis of the BMPR2 gene}

DNA was extracted from $10 \mathrm{ml}$ of whole-blood with Puregene Genomic DNA purification kit (GENTRA systems, Minneapolis, USA) after patient's informed consent was obtained. Exons 1 to 13 of BMPR2 gene were amplified by polymerase chain reaction (PCR) from $100 \mathrm{ng}$ of genomic DNA (Geneamp High Fidelity PCR System Kit, Applied Biosystem, California, USA). The sequence of the primers used has been already reported elsewhere (6). Since we had some difficulties in amplifying exon 12 , we wanted to confirm the data obtained by cDNA synthesis. To this aim, RNA was extracted from peripheral blood cells using TriReagent extraction kit (Sigma Corporation, St Louis, Missouri, USA) and retrotranscribed with the SuperScipt III firstStrand Synthesis System (Invitrogen, Groningen, The Netherlands) using oligo(dt) primers. cDNA was then amplified with the appropriate primers. BMPR2 cDNA was purified with QIAquick gel extraction kit (QIAGEN).

The PCR fragments obtained from genomic DNA and cDNA were sequenced using MWG Biothec service (Ebersberg, Germany) and compared with the published sequence of the gene and of cDNA. We did not find any mutation in the exons 1 to 13 of the BMPR2 gene. 


\section{DISCUSSION}

We report the case of a patient with neurofibromatosis type 1 and PAH. Only seven cases of PAH in von Recklinghausen's disease have been reported so far (9-13). In all reports PAH seems to be related to the vascular involvement of neurofibromatosis although histology was described only in one case.

In our patient all the conditions associated with $\mathrm{PAH}$ such as connective tissue diseases, chronic lung disease, congenital or acquired heart diseases, HIV infection, drug and toxins, hemoglobinopathies, myeloproliferative disorders, thromboembolism were excluded. Vasculopathy associated to neurofibromatosis was the probable cause of $\mathrm{PAH}$, however we wanted to exclude the possibility of mutations in the BMPR2 gene since there was a suspected case of PAH in her grandmother. Indeed in the past year mutations in the BMPR2 gene have been identified in a high proportion of cases of familial and sporadic PAH. BMP type II receptor is located in lipid rafts, including caveolae, of pulmonary endothelium in vivo and in vitro (14). Caveolae are vescicular organelles that are particularly abundant in cells of cardiovascular system including endothelial cells, smooth muscle cells and fibroblasts (4). Caveolin knockout mice display dramatic abnormalities in the cardiovascular system, such as pulmonary hypertension and cardiac hypertrophy (15). Neurofibromin, the protein encoded by the NF1 gene has been detected in the endothelial cell layer of rat cerebral vessels, renal arteries, and aorta by immunohistochemistry. Neurofibromin expression was also detected in smooth muscle layer of the aorta (16). Recently some authors have reported that neurofibromin modulates Akt, an important regulator of cell proliferation and survival, and binds to caveolin-1 (4). Therefore the interplay of neurofibromin, caveolin and akt may play a pivotal role in the pathogenesis of $\mathrm{PAH}$ in patients with NF1.

In conclusion, based on the histological findings of the lung tissue and on the genetic analysis of BMPR2, we think that PAH was a complication of NF1 in our patient and suggest that patients with NF1 need to be evaluated with trans-thoracic echocardiogram for the presence of PAH, even if it has low sensibility to screen initial forms.

\section{REFERENCES}

1. Reynolds RM, Browning GG, Nawroz I, Campbell IW : Von Reckinghausen's neurofibromatosis : neurofibromatosis type 1 . Lancet $361: 1552$ 1554, 2003

2. Hirsch NP, Murphy A, Radcliffe JJ : Neurofibromatosis : clinical presentations and anaesthetic implications. Br J Anaesth 86 : 555-64, 2001

3. Castle B, Baser ME, Huson SM, Cooper DN, Upadhyaya M : Evaluation of genotype-phenotype correlations in neurofibromatosis type 1 . J Med Genet 40 : 1-5, 2003

4. Boyanapalli M, Lahoud OB, Messiaen L, Kim B, Anderle de Sylor MS, Duckett SJ, Somara S, Mikol DD : Neurofibromin binds to caveolin-1 and regulates ras, FAK, and Akt. Biochem Biophys Res Commun 340 : 1200-8, 2006

5. Humbert M, Sitbon O, Simonneau G : Treatment of pulmonary arterial hypertension. $\mathrm{N}$ Engl J Med 351 : 1425-36, 2004

6. Zemin D, Morse JH, Slager SL, Cuervo N, Moore KJ, Venetos G, Kalachikov S, Cayanis E, Fischer SG, Barst RJ, Hodge SE, Knowles JA : Familial primary pulmonary hypertension (gene PPH1) is caused by mutations in the Bone Morphogenetic Protein Receptor-II Gen. Am J Hum Genet 67 : 737-744, 2006

7. Sugiyama S, Hirota H, Yoshida M, Takemura Y, Nakaoka Y, Oshima Y, Kazuhiro T, Izumi M, Fujio Y, Hasegawa S : Novel insertional mutation in the Bone Morphogenetic Protein Receptor type II associated with sporadic primary pulmonary hypertension. Circulation J 68 : 592594, 2004

8. Rudarakanchana N, Morrell NW : Primary pulmonary hypertension : molecular basis and potential for therapy. Expert Rev Mol Med $6: 1$ 15, 2004

9. Samuels N, Berkman N, Milgalter E, Bar-Ziv J, Amir G, Kramer MR: Pulmonary hypertension secondary to neurofibromatosis : intimal fibrosis versus thromboembolism. Thorax 54 : 858-859, 1999

10. Aoki Y, Kodama M, Mezaki T, Ogawa R, Sato M, Okabe M, Aizawa Y : von Recklinghausen disease complicated by pulmonary hypertension. Chest 119 : 1606-1608, 2001

11. Hernandez FJG, Roman JS, Mediana CO, Romero LM : Hipertension arterial pulmonary primaria en un paciente con neurofibromato- 
sis. Med Clin (Barc) $118:$ 78-79, 2002

12. Porterfield JK, Pyeritz RE, Traill TA : Brief clinical report : pulmonary hypertension and interstitial fibrosis in von Recklinghausen neurofibromatosis. Am J Med Genet 25 : 531-535, 1986

13. Engel PJ, Baughman RP, Menon SG, Kereiakes DJ, Taylor L, Scott M : Am J Cardiol 99 : 11771178, 2007

14. Ramos M, Lame MW, Segall HJ, Wilson DW : The BMP type II receptor is located in lipid rafts, including caveolae, of pulmonary endothelium in vivo and in vitro. Vascul Pharmacol $44: 50-59,2006$

15. Li Xa, Everson Wv, Smart EJ : Caveolae, lipid raft, and vascular disease. Trends Cardiovasc Med 15 : 92-6, 2005

16. Norton KK, Xu J, Gutmann DH : Expression of the neurofibromatosis I gene product, neurofibromin, in blood vessel endothelial cells and smooth muscle. Neurobiol Dis $2: 13-21,1995$ 